\title{
Preparedness of Acute Care Facility and a Hospital for COVID-19 Pandemic: What We Did!
}

\author{
Sameer $\mathrm{Jog}^{1}$, Dhananjay Kelkar ${ }^{2}$, Madhav Bhat ${ }^{3}$, Sampada Patwardhan ${ }^{4}$ Purushotham Godavarthy ${ }^{5}$, Ujwal Dhundi ${ }^{6}$, \\ Harshwardhan S Pawar ${ }^{7}$, Prasad Rajhans ${ }^{8}$, Balasaheb Pawar ${ }^{9}$, Vishnudas S Telbhare ${ }^{10}$, Gouri Ranade ${ }^{11}$, Vaibhavi Upadhye ${ }^{12}$, \\ Parikshit S Prayag ${ }^{13}$, Bharat Purandare ${ }^{14}$, Sukrut Purandare ${ }^{15}$, Rajesh Bhavsar ${ }^{16}$
}

\begin{abstract}
Background and aim: India is facing the pandemic of coronavirus disease (COVID-19) just like the whole world. The private sector is the backbone of a healthcare facility in India. Presently, only a few major hospitals in the country are actively dealing with the COVID-19 patients while others are facing troubles due to lack of manpower, management, and required experience to face the pandemic. Despite the lockdown, the cases are ever increasing and each and every hospital in the country should be prepared to face this pandemic the world has never seen before. As one of the largest multispecialty hospitals and a designated COVID center, we have developed and adopted some strategies for better preparedness to face the surge of this pandemic. We would like to share our experience and hope that the strategies laid down and adopted by us will help many other acute care facilities in many parts of India.

Materials and methods: Different strategies are adopted to deal with the crisis situation of the COVID-19 pandemic. Our adopted strategies were directed to mitigate the challenges of administration, hospital space organization, management of staff and supplies, maintenance of standard of care, and specific COVID care and ethics during this pandemic.

Results: Based on strategies adopted by us, we feel more confident and prepared to deal with COVID-19 pandemic.

Conclusion: Our approach for preparing for the COVID-19 pandemic may not be the best one but we believe that the basic managerial principles we adopted will guide many other institutions to find their path in tackling the pandemic in the best possible way.

Keywords: COVID pneumonia, Intensive care unit, Pandemic, Preparedness.

Indian Journal of Critical Care Medicine (2020): 10.5005/jp-journals-10071-23416
\end{abstract}

\section{INTRODUCTION}

India is facing the pandemic of coronavirus disease (COVID-19) just like the whole world. Governments, policymakers, and healthcare institutions all over the world are apprehensive. Private hospitals play an important role in the Indian healthcare system. and many people resort to them for their acute health problems. ${ }^{1}$ Despite the presence of strict legislation and lockdown, the cases of COVID-19 are increasing day by day and a surge in cases is expected after cessation of lockdown. As one of the largest multispecialty hospitals and a designated COVID center, we have developed and adopted some strategies for better preparedness to face the surge of this pandemic. We would like to share our experience and hope that the strategies laid down and adopted by us will help many other acute care facilities in other parts of India.

\section{Framework for Preparing the Hospital for Pandemic}

Based on our hospital's successful experience in the management of the H1N1 pandemic ${ }^{2}$ and the early literature from Italian ICUs, ${ }^{3}$ the hospital administrative authorities designed various policies applicable in the Indian context, for dealing with the COVID-19 pandemic. We have divided our policies under the following headings:

- Administration

- Organization of hospital space

- Staff management

- Supplies
1,5-11 Department of Critical Care and Emergency Medicine, Deenanath Mangeshkar Hospital, Pune, Maharashtra, India

${ }^{2}$ Department of Oncosurgery, Deenanath Mangeshkar Hospital, Pune, Maharashtra, India

${ }^{3}$ Department of Ophthalmology, Deenanath Mangeshkar Hospital, Pune, Maharashtra, India

${ }^{4}$ Department of Microbiology, Deenanath Mangeshkar Hospital, Pune, Maharashtra, India

${ }^{12}$ Department of Post Graduate Certification, Deenanath Mangeshkar Hospital, Pune, Maharashtra, India

${ }^{13}$ Department of Transplant Infectious Diseases, Deenanath Mangeshkar Hospital, Pune, Maharashtra, India

${ }^{14}$ Department of Infectious Disease, Deenanath Mangeshkar Hospital, Pune, Maharashtra, India

${ }^{15}$ Department of Medicine, Deenanath Mangeshkar Hospital, Pune, Maharashtra, India

${ }^{16}$ Department of Cardiology, Deenanath Mangeshkar Hospital, Pune, Maharashtra, India

Corresponding Author: Sameer Jog, Department of Critical Care and Emergency Medicine, Deenanath Mangeshkar Hospital, Pune, Maharashtra, India, Phone: +91 9823018178, e-mail:drsjog@gmail.com How to cite this article: Jog S, Kelkar D, Bhat M, Patwardhan S, Godavarthy P, Dhundi U, et al. Preparedness of Acute Care Facility and a Hospital for COVID-19 Pandemic: What We Did! Indian J Crit Care Med 2020;24(6):385-392.

Source of support: Nil

Conflict of interest: None

(0) The Author(s). 2020 Open Access This article is distributed under the terms of the Creative Commons Attribution 4.0 International License (https://creativecommons. org/licenses/by-nc/4.0/), which permits unrestricted use, distribution, and non-commercial reproduction in any medium, provided you give appropriate credit to the original author(s) and the source, provide a link to the Creative Commons license, and indicate if changes were made. The Creative Commons Public Domain Dedication waiver (http://creativecommons.org/publicdomain/zero/1.0/) applies to the data made available in this article, unless otherwise stated. 
- Maintenance of standard of care

- Specific COVID ICU management

- The trade-off between usual care and pandemic care for nonCOVID patients.

\section{Administration}

Single-point Decision-making-Creating Organization Chart for Pandemic

A senior physician intensivist was made in charge of the task force for policymaking and handling of the pandemic patients in the institution. His responsibilities included the following:

- Liaising with the local, state, and national government authorities

- Time table and manpower management of the ICUs, emergency rooms, and fever clinic

- Disseminating and updating the knowledge of clinical management of the pandemic situation

- Implementation of the polices adapted to Indian standards depending upon the availability of resources for the ICUs as well as to other departments

- Disseminating guidelines over rational use of medical resources in pandemic situations throughout the hospital

- Keeping watch over various departments involved in the implementation of policies related to pandemic management

- Troubleshooting and quick remedial measures in the least possible time.

\section{Division of Responsibilities and Ensuring Accountability}

Various responsibilities in the management of the pandemic situation were assigned to different persons as a single point of contact. This helped us to streamline functioning leading to faster and more efficient decision-making and implementation with greater accountability. Various responsibilities were divided into following divisions and one medical or paramedical person was assigned the responsibility of each division according to skill mapping.

- Medical and nonmedical procurement and its distribution in the hospital

- Management of workforce-nurses and multipurpose workers and nonmedical workforce

- Biomedical waste management

- Staff clinic for COVID-19 (suspected members of hospital staff)

- Staff accommodation and quarantine

- Communication and coordination with government bodies such as a municipal corporation, state, and central health agencies by dedicated officers appointed by the hospital

- Food supply to staff and patients

- Laundry

- Dead body management and handover

- Writing working guidelines and standard operating procedures for medical and surgical departments to prevent Corona Virus infection in non-COVID hospitalized patients

- Print, electronic, and social media management

\section{Cessation of Routine Non-urgent Work}

All non-urgent and elective procedures and OPD visits were stopped. Consultants and patients were encouraged to use telephonic/WhatsApp or video consultations to decrease the footfall in the hospital. Systems and procedures were set for such telephonic/video consultations/WhatsApp group chats, e.g., routine antenatal consultations for pregnant women registered in the hospital (Appendix 1). At the same time, emergency management (medical and surgical) of non-COVID patients was continued without interruption by non-COVID teams, e.g., for acute stroke, acute coronary syndrome, and surgical emergencies.

\section{Use of Social Media for Inside Connectivity}

All the stakeholders within the hospital such as departmental heads and task force for COVID-19 management were connected all the time through a dedicated WhatsApp group to prevent physical interactions and to be regularly updated on day-to-day problems, policies, and their progress and new research findings.

\section{Organization of Hospital Space}

- Due to the highly infectious nature of the disease, the patient care area needed to be divided into two separate zones, the COVID care zone and the non-COVID care zone. We have dedicated one building independent of the main hospital complex, as a stand-alone COVID care area in the hospital. This building included a fever (triage) clinic, emergency room, suspect patient zone, ICU, isolation wards, and rooms (Fig. 1).

\section{Emergency Room}

Divided into two zones, COVID bay and non-COVID bay, one for severe acute respiratory infection (SARI) patients/COVID-19 suspects and the other for non-SARI patients, respectively (Fig. 1).

\section{Fever OPD}

All nonserious patients coming with complaints of fever; symptoms of upper respiratory tract infection like sore throat, cough, cold, etc.; and travel history were directed to the fever clinic.

\section{Dedicated COVID ICU}

The ICU complex having an independent air-exchange unit with dedicated equipment including ventilators, ultrasound machine, and dialysis machine was kept exclusively for COVID-19-positive patients. Dedicated teams were assigned for ICU who were rotated as per the rotational policy.

\section{Isolation Wards}

One hundred sixty-bedded isolation wards and rooms were established to accommodate noncritical COVID-19 patients.

- Dedicated lifts with special cleaning policies were earmarked for transportation of COVID-19 patients from the emergency room and within the hospital for various procedures as required.

- A cluster ward of 30 beds was established for the accommodation of suspected clusters coming either from the community or from within the hospital itself.

- Earmarking of additional areas for COVID care-additional area for fever clinic and other COVID care areas such as ICU, wards, and HDU were identified for expansion of facilities in the event of a rapid surge in the number of cases.

- All major entrances of the hospital were manned by staff who were trained to screen all patients coming into the hospital. The trained staff screened all incoming patients for symptoms 




Fig. 1: Schematic representation of the hospital area for COVID care facility

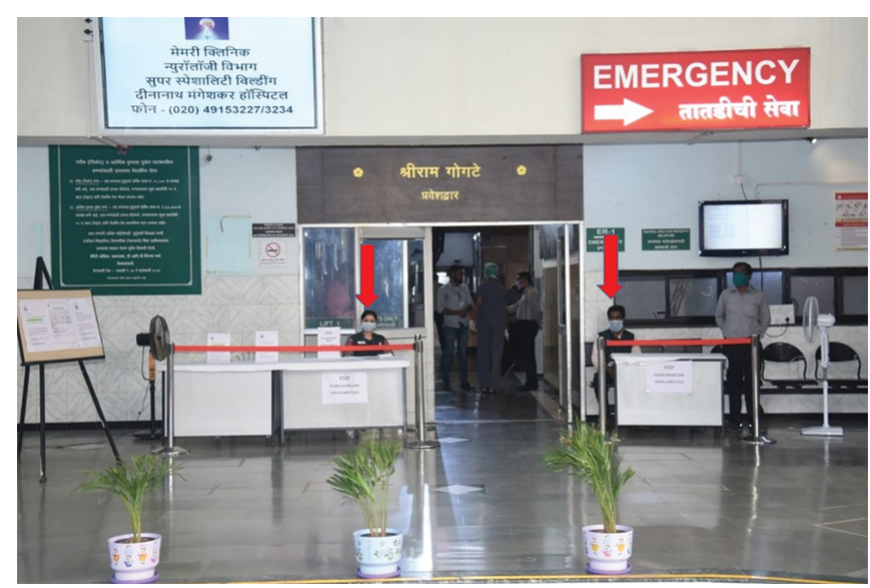

Fig. 2: Manned hospital entrance for patient screening and segregation

such as fever, cough, dyspnea, myalgia, and travel and contact history and directed the patients to the fever clinic if required (Fig. 2, arrows).

\section{Staff Management and Empowerment}

Certain issues related to human resources were addressed by the hospital management and staff was empowered for better handling of a stressful situation like a highly infectious disease. Some of the issues were addressed as follows:

\section{Formation of COVID-19 Team}

A dedicated COVID-19 team was formed that consisted of experienced staff who had witnessed the H1N1 pandemic in the past and motivated younger staff to balance the staff ratio. This team included nurses, doctors, and other healthcare workers (HCWs) in the hospital. The management of the initial few patients by a team led by senior intensivists helped to build confidence in the staff. Young HCWs ( $<45$ years of age) were preferably recruited as they were less likely to get a severe infection. ${ }^{4-6}$ Also, HCWs including senior consultants with age more than 60 and those on immunosuppressive therapy or having multiple comorbidities were not included in the COVID-19 team.

\section{Duties and Rotation Policy}

Duties were allocated in a centralized manner giving equal chances to everyone. This was meant to prevent any bias in the allocation of duties. We divided our staff including doctors, nurses, respiratory therapists, and ground staff in several groups. Each group was rotated in the COVID-19 care area depending upon their skills, i.e., in ICU, isolation wards, and fever clinic. Each team worked in the COVID care area for a week followed by 1 week off as a cooling time. Other teams during the same week period were divided into a buffer team that worked in the non-COVID-19 care area. A separate duty roster was prepared, both for general care staff and COVID care staff every week.

\section{Motivation}

The positive attitude of HCWs was enhanced to prevent dropouts. This was done by regular interaction and motivation by the chief intensivist. In the hospital, accommodation was provided for staff with smaller houses and larger families during the duty and the cooling period. This helped to keep the staff grounded and feel cared for and valued. This became evident when 38 nurses, 12 multipurpose workers, and 3 teams of ICU and emergency room doctors volunteered for work in the COVID team even before the first patient had arrived on March 22, 2020.

\section{Rotation and Cooling-off}

Each team was posted in the COVID care area for a week followed by a week off as a cooling-off period and isolation measures. After the cooling-off period of 1 week, this team is posted in the non-COVID area for a minimum of 7 days. This rotation policy ensured that no team was posted in the COVID care area for greater than 7 days at a time within 21 days. This helped us to prevent the burnout of staff working in the COVID care area continuously and ensure maximum safety of staff without compromising the care in COVID-19 patients.

\section{Leave Policy}

There was a reduction in ICU and hospital workload following directions issued by the Government of India to stop all elective procedures from the 2 nd week of March. The rotational leave policy was designed to use the staff as a buffer during need in the event of a rapid surge, reduce exposure of HCWs to COVID-19, and also to compensate for extra duties during the surge. 


\section{Healthcare Worker Chemoprophylaxis}

Chemoprophylaxis with hydroxychloroquine tablets on hospital expense was provided by the hospital to all HCWs dealing with COVID-19 cases as per the recommendations of the Indian Council of Medical Research (ICMR). ${ }^{7}$

\section{Backup Buffer of Manpower}

A separate standby duty roster of registrars and consultants for COVID ICU was planned as an emergency backup buffer in case the primary team members fell ill or needed emergency leave for any other purpose and to prevent an unforeseen shortage of manpower.

Expansion of workforce: Given the possibility of surge in patient number, we are training residents and doctors in all specialties to work in COVID and non-COVID area as per their skills so that they can be deployed when necessary.

\section{Sanitization and Isolation}

Clean scrubs were provided to all staff working for more than 4 hours on the campus. Shower facility and accommodation were provided by the hospital to staff if they wished to stay back during the COVID ICU duty.

\section{Knowledge of Resources and Updates}

- Ready-to-use documents, posters, and videos regarding precautions and standard operating procedures were displayed on hospital intranet and also at multiple prominent sites in the hospital.

- The FAQs containing details about COVID were posted on hospital intranet and were regularly updated to enable HCWs to gather real-time information from reliable sources. This helped them to follow uniform guidelines obtained from authentic sources with a simple search and prevent deviations and irregularities.

\section{Surveillance of Healthcare Worker}

- Advanced declaration of any overseas travel or exposure by HCWs and their compulsory quarantine was practiced during the initial phase of the pandemic.

- Dedicated staff clinic manned by senior physicians $24 \times 7$ for early detection of potential COVID-19 in hospital staff was established.

- Self-reporting of fever and respiratory symptoms was encouraged among all staff members.

\section{Addition of COVID-related Protocols to Existing Standard of Practices (SOPs)}

Dedicated COVID-19-related standard of practices (SOPs) were prepared in consultation with different departments based on the potential risk of transmission of a novel coronavirus in different situations and procedures, e.g., cath lab, delivery rooms, endoscopy rooms, intubations, and extubations. All SOPs were viewed and modified to comply with the requirements concerning COVID-19 care

\section{Education and Simulation}

Education and simulation of the scenarios that were likely to be faced helped to improve confidence and actual care delivery in a crisis. Keeping this in mind, we repeatedly took education and simulation workshops for different levels of hospital staff.
Multiple simulation workshops were conducted by senior intensivists and a simulation specialist in small batches following the rule of social distancing. The education and simulation workshops covered the following issues:

- Techniques of donning and doffing of personal protective equipment (PPE)

- Hand hygiene techniques

- N95 mask wearing and seal check

- Role-playing of an airway assistant in various hospital scenarios that could be encountered in COVID19 ICUs, e.g., cardiopulmonary resuscitation, patient transport, and accidental extubation

- Newly recommended practices with perceived benefits such as intubations using video laryngoscopes, use of the acrylic aerosol box, etc.

- Checklists for preparation of drugs and equipment trolley for various procedures like intubation, central line, Foleys, etc., to minimize staff movement and enhance efficiency

\section{COVID-19 Patient's Admission and Care Rights}

The hospital assigned exclusive admission rights to three infectious disease specialists for noncritical cases and five intensivists for ICU admission of COVID suspects and confirmed cases. All suspected and confirmed patients with COVID-19 were admitted under this team.

All the clinicians were advised to direct all suspected or confirmed patients of COVID-19 to these designated COVID-19 team physicians/intensivists for further management. No other physician on the hospital panel was allowed admission rights for such patients. Also, screening of the patients admitted to other subspecialties, if needed, was done by one of these three physicians. The decision of the designated COVID care physicians and intensivists regarding isolation and testing was deemed as final as per the hospital policy.

This helped us to reduce exposure of many physicians to the virus and to properly channelize the pandemic care. It avoided unnecessary chaos and unnecessary admissions of patients due to fear in physicians and surgeons of other specialties. We also believed that this policy allowed us to rationally utilize the available resources.

\section{Avoidance of Cross-contamination and Infection among Healthcare Workers}

- Healthcare workers' health is of prime importance as it may affect the health as well as the morale of other HCWs too. Infection of HCWs during the pandemic will lead to a serious loss to the hospital in terms of loss of manpower and also affect the morale of others. ${ }^{8}$ Therefore, utmost precautions were taken to encourage social distancing at workplace like avoiding in-person meeting at the administration level, strict orders of not to gather during the hospital stay for tea or meals, etc. Avoidance of the use of common areas like the cafeteria and lifts was encouraged. Strong emphasis was given on hand hygiene.

Till April 26, 2020, the hospital had cared for 50 COVID-19 patients. A total of $264 \mathrm{HCWs}$ had worked for 7 consecutive days in COVID areas and none of them developed COVID-19 infection. This success could be attributed to proper precautions and training. 
- Admissions of the suspected patients were strictly done in designated areas avoiding any mixing up with other nonsuspect patients. All patients admitted to the hospital were given a mask to cover the nose and face compulsorily.

- Patients tested negative were immediately shifted from the isolation area to non-COVID area.

- A senior and more experienced physician/intensivist on duty was preferred for performing an aerosol-generating procedure to ensure the smooth and rapid performance of the procedure, thus minimizing risk to all HCWs.

\section{Preparing Death Protocol}

Disposal of patients brought dead to the emergency room was done based on guidelines issued by ICMR, Pune Municipal Corporation, and state government rules. Paperwork included Form C, death form, information to government authorities, body preparation, and disposal with municipal authorities. ${ }^{9}$

\section{Supplies}

The need assessment was carried out to identify essential equipment, medications, and other medical supplies that would be needed for the care of COVID-19 patients as well as for prophylaxis measures. Special attention was given to ensure adequate supply by daily updating the stock list of the same as given below (Appendix 2).

\section{Procurement and Rational Use}

Medical equipment, drugs, and other supplies

- Inventory of existing medical equipment was carried out including:

- Ventilators

- Defibrillators

- Backup power supply

- Adequate battery backup for the transportation of patients

- Oxygen supplies

- Critical drugs

- Specific requirements like PPE, N95 filtering face piece respirator (FFR), etc., were identified by the pharmacy.

Emergency procurement of equipment-A dedicated team was established for the procurement of equipment/drugs/sanitizers based on the projected requirement.

\section{Liaison}

Was carried with various government, private agencies, and other hospitals for procurement of medical supplies.

\section{Local Production}

We started in-house production of alcohol-based hand disinfectants to mitigate issues of cost and availability, given its very high consumption.

\section{Overcoming the Resource Limitations}

Rationalizing COVID-19 testing and limitation of indiscriminate use: Considering the possibility of indiscriminate use of nasal and oropharyngeal swabs for even nonsuspect patients admitted to the hospital for other reasons, a testing policy was formulated to test only those patients recommended by designated ID specialists and intensivists.

N95 mask extended use and reuse: ${ }^{10}$ We encouraged the practice of extended use of the $\mathrm{N} 95$ respirator mask for repeated close contact encounters of several patients, e.g., in the emergency room, screening area, fever clinic, ICU, and isolation wards.

We initially planned to adopt the five FFR per individual use policy suggested by the CDC for reuse. However, we were forced to adapt to a strategy that included decontamination and reuse of FFR as per recent research and FDA's emergency use authorization on decontamination of FFR in absence of manufacturer's recommendation. ${ }^{11}$ Out of three methods found to be useful, i.e. ultraviolet germicidal irradiation, vaporous hydrogen peroxide (plasma sterilization), and moist heat, we adopted the plasma sterilization technique.

Special instruction for the reuse of decontaminated FFR was issued to the hospital staff utilizing it as per recommendation by CDC.

All FFRs used during the aerosol procedures were discarded and not reused.

Rationalization of the proper use of PPE: The PPE is the backbone of the safety of HCWs during patient management. In pandemic situations where a large number of cases are expected, efforts should be made to use every bit of PPE rationally. There should not be an unnecessary use of PPE for nonindicated uses and at the same time, appropriate PPE should be available for indicated high-risk situations. Therefore, we came up with a strict PPE policy for every individual of the hospital staff. This is based on CDC recommendations on pandemic guidelines, modified as per hospital requirements and resources. This was mandated by resource-limited settings in India. There was already a dearth of supply of PPEs and also considering that the pandemic would probably last for the next few months, there were fewer chances that the supply of PPEs would sustain for the entire period. Hence, the hospital came up with a self-sustainable plan. This included:

- Full PPE-Commercially available full PPE kit was reserved for those who were involved in aerosol-generating procedures such as swab collection, intubation, extubation, and bronchoscopy only.

- Modified PPE (cap, N95 mask, gown with waterproof aprons, goggles, gloves)-for staff, doctors, and cleaners in COVID-19 ICU for routine care (Fig. 3).

- Surgical mask-All doctors, nurses multipurpose workers (MPWs), technicians coming in physical contact with patients in non-COVID19 areas.

- N95 FFR-Acute COVID bay, fever clinic, isolation room, COVID ICU, medicine OPD doctors, chest OPD doctors, ID doctors.

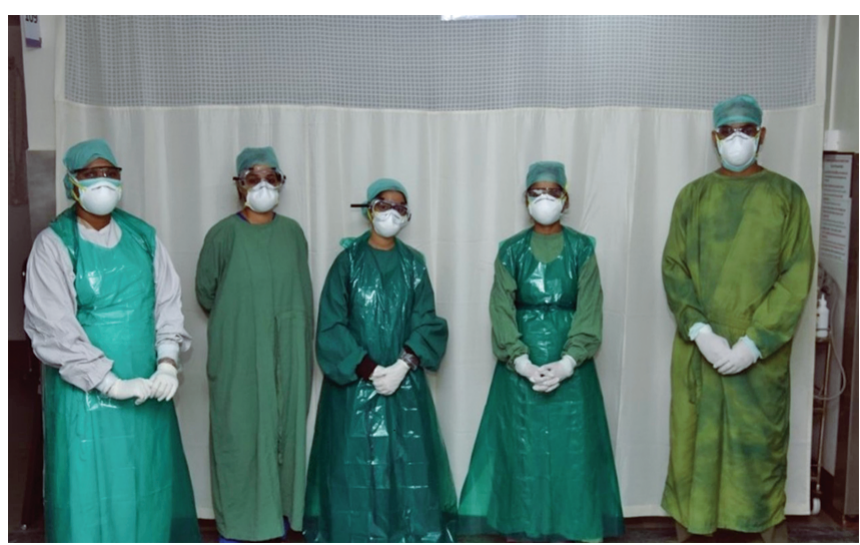

Fig. 3: Modified personal protective equipment 
- Cotton mask (fomite protection) - Staff not in direct contact with patients and in non-COVID-19 areas-receptionist, secretaries, security, accounts, billing, etc. Two such masks were provided-to be washed and reused.

- Goggles and N95 FFRs were being reused after plasma sterilization as per guidelines by CDC.

Negative pressure isolation rooms were not available; therefore, we were cohorting all suspects in private rooms and confirmed cases into the other wards and rooms, thus modifying guidelines to fit available resources.

Acrylic aerosol box-Considering the shortage of acrylic aerosol boxes, transparent drapes were used to cover the patient while performing procedures like intubation, extubation, and bronchoscopy to prevent dispersion of aerosol.

\section{Maintenance of Standard of Care}

Since the pandemic was likely to progress and the ICU care was likely to migrate from conventional to contingency to crisis mode, precautions needed to be taken to prevent the dilution of the standard of care. To minimize the dilution, uniform protocols for ICU care were prepared and practiced in advance including intubation, extubation protocols, sedation, ventilatory management, diet, investigations, etc. The same could be implemented as a standard of care without any confusion with reduced/less experienced manpower as per the availability.

\section{Specific COVID ICU Management}

- Motivation and team building of intensivists

- Allocation of various roles such as preparation of SOPs, training, and simulation to different dedicated intensivists
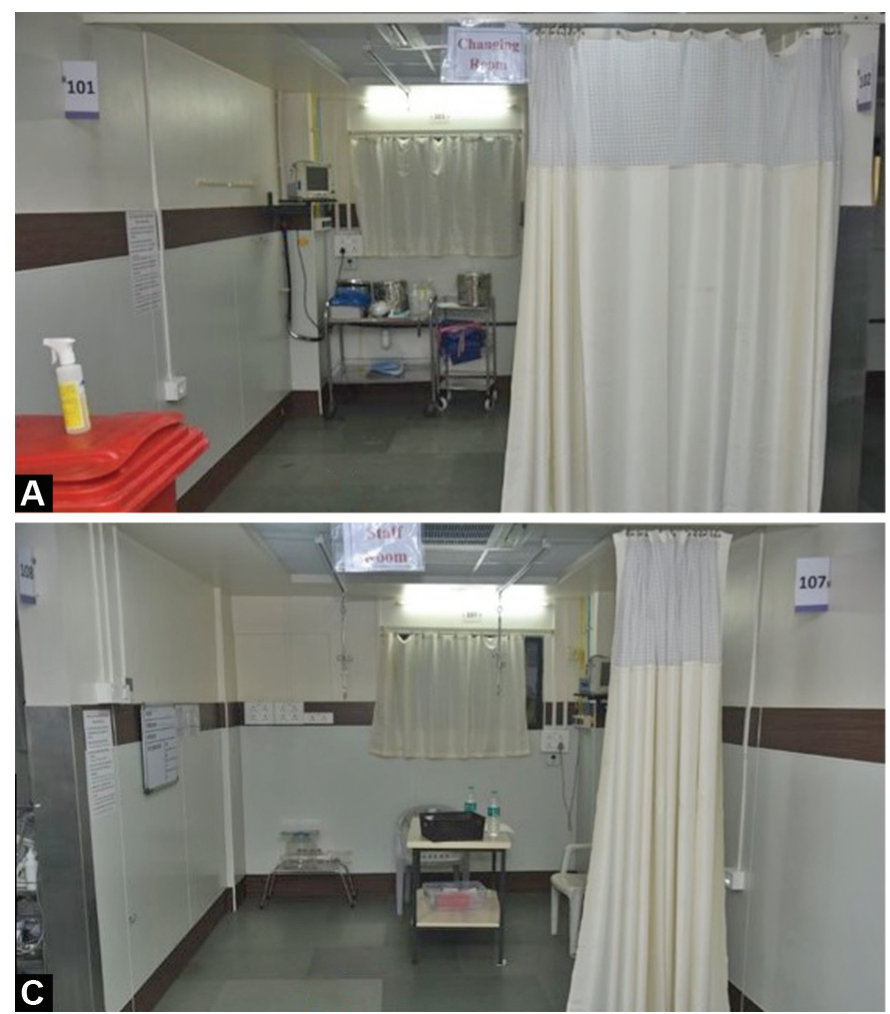

- All nurses and MPWs were briefed by the chief intensivist and departmental heads about the disease and its progress. Realistic and first-hand information of the disease published in journals like NEJM, JAMA, and Lancet as well as by national government bodies was briefed to all HCWs from time to time. This reduced unnecessary anxiety and kept them motivated.

- Staff working in ICU was trained in various skills apart from patient care like shooting chest X-rays, ECHO evaluation, chest physiotherapy, etc., to minimize the need of these ancillary HCWs in COVID ICU and reduce unnecessary exposure.

- Dedicated bins for segregation and disposal of biomedical waste generated in the ICU were stationed.

- All registrars/consultants working in ICU were trained on point of care ultrasound (POCUS) to minimize chest X-rays.

- The rights of patients and visitors were modified according to the pandemic law and the same included in the consent for ICU admission.

- The separate donning-doffing areas were created within the dedicated ICU complex (Figs 4A and B).

- A list of anticipated aerosol-generation procedures (AGPs) was prepared and full PPE was used for the above procedures as mentioned earlier. Routine ICU care was carried out using a protective gown, N95 mask, and protective goggles and gloves.

- The dedicated area for resting and refreshment was earmarked - within the ICU premises itself (Fig. 4C).

- A separate table outside the ICU was placed for supplies (Fig. 4D).

- The provision of meals was made by the hospital within the ICU complex for staff posted in the ICU.

- Unnecessary AGPs were avoided such as mouth care with toothbrushing, nebulization, etc. In the case of special needs, metered-dose inhalers with spacers were utilized to deliver the medicine.

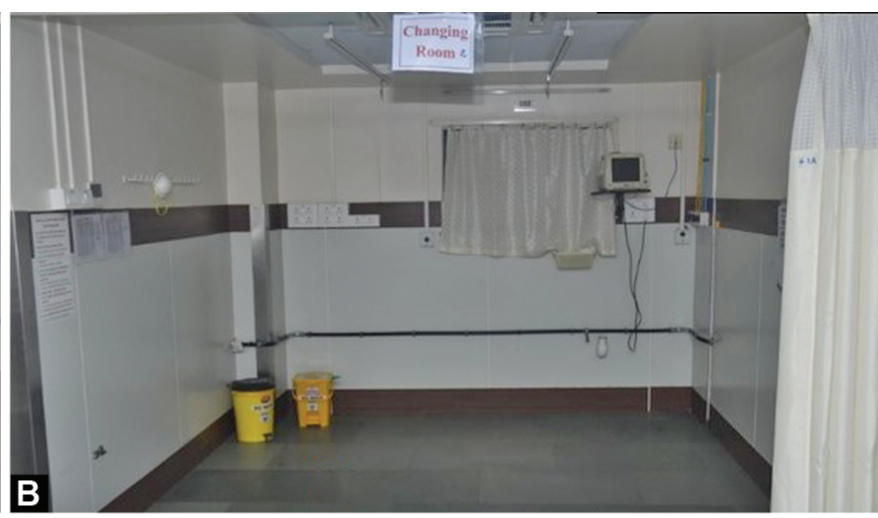

Figs 4A to D: (A) Donning area; (B) Doffing area; (C) Refreshment area; (D) Table for sharing 


\section{Trade-off between Usual Guidelines-based Care and Pandemic Care}

- Mandatory screening of all visitors to ICU

- Restricted visitor entry

- No stay policy for relatives

- Hospital visitor logs for contact tracing

- The utilization of modern modes of communication like WhatsApp, videoconferencing for the patient, and relative counseling was increased and in-person counseling sessions were discouraged

- Single consent on admission to ICU for COVID-19 patients that included consent for intubation, mechanical ventilation support, invasive line placement, prone ventilation, renal replacement therapy, and pandemic disclaimer alert on clinical notes to inform the limitations of routine healthcare delivery due to pandemic situation to avoid unnecessary conflicts (Appendix 3).

\section{Results}

Based on strategies adopted by us, we feel more confident, resourceful, and better prepared to deal with the COVID-19 pandemic.

\section{CONCLUSION}

Pandemics are best fought with appropriate management of manpower, equipment, supplies, and knowledge. But above all, it is the planning and rationalization of the use of resources in resourcelimited settings that are the most important components of our preparations. Repeated simulation and drills along with repeated motivation by seniors help in increasing the confidence of HCWs. Our approach for preparing for the pandemic may not be the best but we believe that outlining the basic managerial principles we adopted will guide many other institutions to find their path in tackling the pandemic in the best possible way.

\section{References}

1. Government of India MoHaFW. NATIONAL FAMILY HEALTH SURVEY (NFHS-4) 2015-16. 2017.

2. Jog S, Kadam D. H1N1 influenza pandemic: What we did and what we learnt? Indian J Crit Care Med 2013;17(5):271-272. DOI: 10.4103/09725229.120316.

3. Cecconi MPA, Grasselli G. Shared experience \& guidance from our colleagues in Northern Italy Milan 2020 [cited 202023 April]. Available from: https://www.esicm.org/covid-19-update-from-our-colleaguesin-northern-italy/.

4. Guan W-J, Ni Z-Y, Hu Y, Liang W-H, Ou C-Q, He J-X, et al. Clinical characteristics of coronavirus disease 2019 in china. New Eng J Med 2020(18). DOI: 10.1056/NEJMoa2002032.

5. Onder G, Rezza G. Brusaferro S. Case-fatality rate and characteristics of patients dying in relation to COVID-19 in Italy. JAMA 2020. DOI: 10.1001/jama.2020.4683.

6. McMichael TM, Currie DW, Clark S, Pogosjans S, Kay M, Schwartz NG, et al. Epidemiology of COVID-19 in a long-term care facility in king county, Washington. New Eng J Med 2020. DOI: 10.1056/ NEJMoa2005412.

7. Research IcoM. Recommendation for empiric use of hydroxychloroquine for prophylaxis of SARS-CoV-2 infection. 2020.

8. Sim MR. The COVID-19 pandemic: major risks to healthcare and other workers on the front line. Occup Environ Med 2020;77(5):281-282. DOI: 10.1136/oemed-2020-106567.

9. Government of India MoHFW, Directorate General of Health Services COVID-19:Guidelines on dead body management. 2020.

10. Centre for disease control and prevention. C:: "Questions and Answers Regarding Respiratory Protection For Preventing 2009 H1N1 Influenza Among Healthcare Personnel" [April 17, 2020]. Available from: https://www.cdc.gov/niosh/topics/hcwcontrols/ recommendedguidanceextuse.html.

11. prevention Cfdca. Decontamination and Reuse of Filtering Facepiece Respirators 2020 [April 18,2020]. Available from: https://www.cdc. gov/coronavirus/2019-ncov/hcp/ppe-strategy/decontaminationreuse-respirators.html. 


\section{Appendix 1: WhatsApp Group for Pregnant Patients for Solving their Problems}

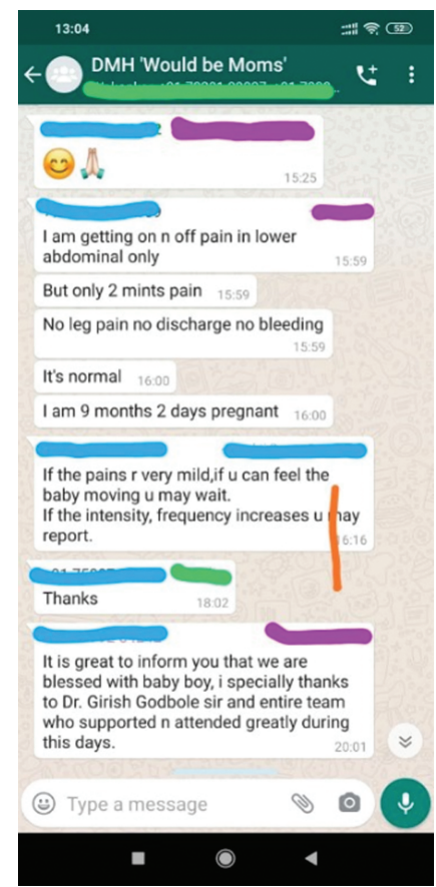

\section{Appendix 3: COVID Pandemic Alert}

- Routine standard of care cannot be given during COVID-19 pandemic period.

- Modified decisions may not be the best in normal circumstances, yet will be implemented based on available data and guidelines across the world.

- Deficiencies arising out of this unprecedented situation have to be accepted by patients and doctors alike.

\section{Appendix 2: Daily Updated Stock List}



